\title{
Cosmological Evolution of Pilgrim Dark Energy
}

\author{
M. Sharif *and M. Zubair ${ }^{\dagger}$ \\ Department of Mathematics, University of the Punjab, \\ Quaid-e-Azam Campus, Lahore-54590, Pakistan.
}

\begin{abstract}
We study pilgrim dark energy model by taking IR cut-offs as particle and event horizons as well as conformal age of the universe. We derive evolution equations for fractional energy density and equation of state parameters for pilgrim dark energy. The phantom cosmic evolution is established in these scenarios which is well supported by the cosmological parameters such as deceleration parameter, statefinder parameters and phase space of $\omega_{\vartheta}$ and $\omega_{\vartheta}^{\prime}$. We conclude that the consistent value of parameter $\mu$ is $\mu<0$ in accordance with the current Planck and WMAP9 results.
\end{abstract}

Keywords:Dark energy; Cosmological parameters.

PACS: 95.36.+x; 98.80.-k; 04.50.Kd.

\section{Introduction}

Over the past decade, the substantial progress in astronomical observations indicate that our universe is presently going through the phase of accelerated expansion. Observations of type Ia supernovae (SNeIa) (Perlmutter et al. 1999; Riess et al. 2007), anisotropy measurement in current cosmic microwave background (CMB) from WMAP (Spergel et al. 2004) and data of

*msharif.math@pu.edu.pk

†mzubairkk@gmail.com 
large scale structure (LSS) from Salon Digital Sky Survey (SDSS) (Tegmark et al. 2004) strongly endorse this manifestation. The mechanism behind the expanding paradigm is usually assigned to exotic energy component with strong negative pressure entitled as dark energy (DE). This may determine the ultimate future of the universe but its cosmological origin and characteristics are still a complicated story. The most likely theoretical campaigner of $\mathrm{DE}$ is the cosmological constant $\Lambda$ with equation of state (EoS) $\omega_{\vartheta}=-1$ (Weinberg 1989; Peebles and Ratra 2003). The model comprising of $\Lambda$ and cold dark matter (CDM) dubbed as $\Lambda$ CDM model which suffers from fine tuning and cosmic coincidence puzzles. However, the predictions of $\Lambda \mathrm{CDM}$ model appear to fit the current observational data (Samushia and Ratra 2008; Jassal et al. 2008). Numerous candidates of DE have been suggested in literature such as quintessence $\left(\omega_{\vartheta}>-1\right)$, phantom $\left(\omega_{\vartheta}<-1\right)$ violating the null energy condition, quintom with $\omega_{\vartheta}$ evolving across -1 , K-essence, tachyon, ghost condensate, holographic DE (HDE) and so forth (Arkani-Hamed et al. 2002; Armendariz-Picon et al. 2000; Caldwell 2002; Feng et al. 2005; Hsu 2004; Li 2004; Steinhardt et al. 1999). Introducing new ingredients of DE to the whole cosmic energy is one way to handle the issue of cosmic acceleration. Another approach is the modification of Einstein Lagrangian to get modified theories such as $f(R)$ (Sotiriou and Faraoni 2010), $f(R, T)$ (Harko et al. 2011; Sharif and Zubair 2012a, 2012b, 2013a, 2013b, 2013c) and $f\left(R, T, R_{\mu \nu} T^{\mu \nu}\right)$ (Haghani et al. 2013; Sharif and Zubair 2013d, 2013e) gravities, $T$ is the trace of the energy-momentum tensor.

Cohen et al. (1999) set up a relation between ultraviolet (UV) and infrared (IR) cut-offs due to the limit made by the formation of a BH. If $\rho_{\vartheta}$ is the quantum zero-point energy density associated with UV cut-off then entire energy in a sysytem of size $L$ should not exceed BH mass of the same size so that $L^{3} \rho_{\vartheta} \leqslant L M_{p}^{2}, M_{p}=1 / \sqrt{8 \pi G}$ is the reduced Planck mass. The largest IR cut-off saturates the inequality and one gets the HDE density

$$
\rho_{\vartheta}=\frac{3 c^{2} M_{p}^{2}}{L^{2}}
$$

where $3 c^{2}$ is a numerical constant. Several proposals have been suggested for IR cut-off including Hubble, particle and event horizons as well as conformal age of the universe, Ricci scalar and Granda Oliveros cut-off (Li 2004; Gao et al. 2009; Granda and Oliveros 2008, 2009; Wei and Cai 2008a).

Phantom form of $\mathrm{DE}(\rho+p<0)$ possesses a peculiar feature of big rip, the innumerous cosmic expansion within finite time. In such scenario, 
the energy density grows quickly and disrupts all the large structures and bounded objects. A question arises about the fate of BHs in the universe dominated by phantom DE. One can say that repulsive force would be strong enough to avoid the gravitational collapse and formation of BHs. Babichev et al. (2004) explored phantom energy accretion of $\mathrm{BH}$ and found that its mass decreases gradually. Some authors (Jamil and Qadir 2011; Sharif and Abbas 2011, 2012) discussed this issue for different BH solutions. Gao et al. (2008) showed that physical mass of BH may rather increase due to accretion of phantom energy implying the violation of cosmic censorship conjecture.

Gonzalez and Guzman (2009) tested the accretion of phantom scalar field into $\mathrm{BH}$ with different initial configurations and found that this mechanism can reduce one half of the BH area. Sun (2009) studied dynamical equation of $\mathrm{BH}$ mass in terms of cosmological parameters and obtained that $\mathrm{BH}$ mass reduces to zero for the phantom dominated universe approaching to big rip. Recently, Wei (2012) proposed a new model of DE named as pilgrim DE (PDE) based on the idea that phantom DE is strong enough to avoid the formation of BH. He considered Hubble horizon as an IR cut-off and developed constraints on PDE using the latest cosmic observations. Sharif and Jawad (2013) analyzed the interacting PDE models in terms of present day values of cosmographic parameters.

This paper explores the cosmological evolution of PDE for three cut-offs namely particle horizon, event horizon and conformal age of the universe in FRW universe. We follow the work of $\operatorname{Li}(2004)$ in HDE to explore the features of non-interacting PDE for these cut-offs through fractional DE density $\Omega_{\vartheta}$, EoS parameter $\omega_{\vartheta}$, statefinder diagnostic parameters and $\omega_{\vartheta}-\omega_{\vartheta}^{\prime}$ analysis. The paper has the following format. In next section, we comprehensively present the evolutionary paradigm of PDE. We conclude our results in the last section.

\section{Pilgrim Dark Energy}

The pilgrim dark energy is defined through the relation Wei (2012)

$$
\rho_{\vartheta}=3 n^{2} M_{p}^{4-\mu} L^{-\mu} .
$$

The first Friedmann equation is given by

$$
3 M_{p}^{2} H^{2}=\rho,
$$


where $\rho=\rho_{M}+\rho_{\vartheta}$ comprises of matter as well as DE components and $H$ is the Hubble parameter. The matter energy density is defined as $\rho_{M}=$ $\rho_{M 0} e^{-3 x}(x=\ln a)$ from the matter energy conservation equation. By setting the fractional energy densities of matter and DE

$$
\Omega_{M}=\frac{\rho_{M}}{\rho_{c r i}}, \quad \Omega_{\vartheta}=\frac{\rho_{\vartheta}}{\rho_{c r i}}, \quad \rho_{c r i}=3 M_{p}^{2} H^{2},
$$

Eq.(2) can be cast to the form

$$
\Omega_{m}+\Omega_{\vartheta}=1
$$

or

$$
H(x)=H_{0}\left(\frac{\Omega_{M 0} e^{-3 x}}{1-\Omega_{\vartheta}}\right)^{1 / 2} .
$$

If $\Omega_{\vartheta}$ is known then one can determine the whole expansion history $H(x)$. We discuss cosmological evolution for different cut-offs such as particle and event horizons as well as conformal age of the universe.

\subsection{Particle Horizon}

This horizon was initially used by Fischler and Susskind (1998) in holographic cosmology. Li (2004) discussed HDE by taking particle horizon as an IR cutoff and found that it does not imply realistic cosmology with EoS $\omega_{\vartheta}>-1 / 3$. The particle horizon is defined as

$$
L=R_{p}=a(t) \int_{0}^{t} \frac{d \hat{t}}{a(\hat{t})}=a(t) \int_{0}^{a} \frac{d a^{\prime}}{H a^{\prime 2}} .
$$

Combining the definition of PDE (1) and particle horizon (5), it follows that

$$
\int_{0}^{t} \frac{d \hat{t}}{a(\hat{t})}=\int_{0}^{a} \frac{d a^{\prime}}{H a^{\prime 2}}=\frac{1}{a}\left(\frac{n^{2} M_{p}^{2-\mu}}{H^{2} \Omega_{\vartheta}}\right)^{1 / \mu} .
$$

Equation (3) can be represented as

$$
\frac{1}{H a}=\frac{\sqrt{a\left(1-\Omega_{\vartheta}\right)}}{H_{0} \sqrt{\Omega_{m 0}}} .
$$


Substituting this relation in Eq.([6), we have

$$
\int_{0}^{a} \sqrt{a\left(1-\Omega_{\vartheta}\right)} d \ln a=e^{(3 / \mu-1) x}\left(\frac{n^{2} M_{p}^{2-\mu}}{H_{0}^{2-\mu} \Omega_{m 0}^{1-\mu / 2}}\right)^{1 / \mu}\left(\frac{1}{\Omega_{\vartheta}}-1\right)^{1 / \mu} .
$$

Differentiating it with respect to $x=\ln a$, it follows that

$$
\Omega_{\vartheta}^{\prime}=\Omega_{\vartheta}\left(1-\Omega_{\vartheta}\right)\left(3-\mu-\frac{\mu}{C}\left(1-\Omega_{\vartheta}\right)^{1 / 2-1 / \mu}\left(\Omega_{\vartheta}\right)^{1 / \mu} e^{(3 / 2-3 / \mu) x}\right),
$$

where $C=\left(\frac{n^{2} M_{p}^{2-\mu}}{H_{0}^{2-\mu} \Omega_{m 0}^{1-\mu / 2}}\right)^{1 / \mu}$ and prime indicates derivative with respect to $x=\ln a$. This result can explain the cosmic evolution according to PDE with particle horizon.

One can exactly solve the above equation to represent the behavior of PDE (Li 2004). The corresponding EoS parameter can be set by using the energy conservation equation as

$$
\omega_{\vartheta}=-1-\frac{1}{3} \frac{d \ln \rho_{\vartheta}}{d x}=-1+\frac{\mu}{3}\left(1-\frac{1}{H}\left(\frac{H^{2} \Omega_{\vartheta}}{n^{2} M_{p}^{2-\mu}}\right)^{1 / \mu}\right) .
$$

If we set $\mu=2$, Eqs.(17) and (8) reproduce the corresponding results in HDE with particle horizon as an IR cut-off (Li 2004). For HDE, EoS is $\omega_{\vartheta}=$ $-1+\frac{2}{3 n} \sqrt{\Omega_{\vartheta}}$ indicating that when $\Omega_{\vartheta} \rightarrow 1$ in the future, $\omega_{\vartheta}=-1+\frac{2}{3 n}>\frac{-1}{3}$ which appears to be inconsistent with the accelerating phase. In case of PDE, we have dependence on parameter $\mu$ resulting in extra degrees of freedom. As $n^{2}$ is involved in Eqs.(7) and (8), so the expansion history is independent of signature of $n$. Solving Eq.(17) with the initial condition $\Omega_{\vartheta 0}=1-\Omega_{m 0}$ and using in Eq.(8), the evolution of PDE is shown in Figures 1-4. Here, present day values of $\Omega_{m}$ and $H$ are defined from recent Planck results as $\Omega_{m 0}=0.315$ and $H_{0}=67.3$. The Planck and WMAP9 observations set the constraints for EoS of DE $\omega_{\vartheta}$ as $\omega_{\vartheta}=-1.13_{-0.10}^{+0.13}$ and $-1.71<\omega_{\vartheta}<-0.34$ respectively (Ade et al. 2013)

For $\mu \geqslant 3$, we have purely matted dominated phase of the universe as shown in Figure 1. This choice should be neglected in search of some observationally consistent models. If $\mu<0$, the evolution of $\omega_{\vartheta}$ and $\Omega_{\vartheta}$ are shown in Figure $\mathbf{2}$ which indicates that $\omega_{\vartheta}$ is always in phantom region and never intersects the phantom divide line $\left(\omega_{\vartheta}=-1\right)$ in entire cosmic evolution. This behavior is similar to the case of PDE with Hubble horizon in 

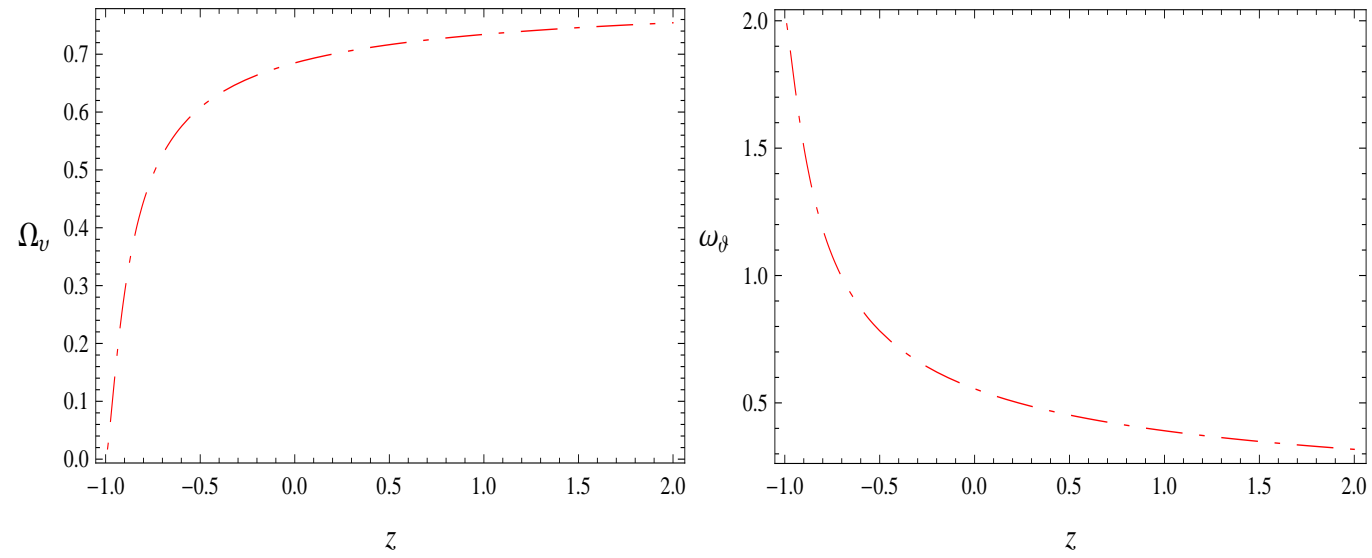

Figure 1: Evolution of $\Omega_{\vartheta}$ and $\omega_{\vartheta}$ for PDE with particle horizon. Here we set $\mu=3$ and $n=2$. It clearly shows that such choice is no more realistic resulting matter dominated regime in the future evolution.

which $\omega_{\vartheta}<-1$ in whole cosmic history (Wei 2012). For the Hubble horizon, $\omega_{\vartheta}$ asymptotically goes to -1 , i.e., it represents de Sitter phase in late times whereas in our case for PDE with particle horizon, it ends up with phantom phase.

The deceleration parameter is defined in terms of $\omega_{\vartheta}$ and $\Omega_{\vartheta}$ as

$$
q=-\frac{a \ddot{a}}{\dot{a}^{2}}=\frac{1}{2}\left(1+3 \omega_{\vartheta} \Omega_{\vartheta}\right),
$$

which is a handy tool to explain the transition from decelerated phase to accelerating regime. We plot $q$ versus $z$ and show the transition from decelerated phase to accelerated era. The universe entered in accelerated era in recent past and it will finish with $q<-1$ representing the phantom evolution. The change of signature in $q$ depends upon the values of $\mu$ and the era of accelerated expansion begins earlier for large values of $\mu$. Differentiating Eq.(8) with respect to $x=\ln a$, we get

$$
\omega_{\vartheta}^{\prime}=\left(1-\frac{3}{\mu}\left(1+\omega_{\vartheta}\right)\right)\left(-1+\frac{\mu}{2}+\frac{1}{3} \frac{d}{d x} \ln \Omega_{\vartheta}\right) .
$$

Caldwell and Linder (2005) discussed the quintessence feature of DE candidate and analyzed its representation in $\omega_{\vartheta}-\omega_{\vartheta}^{\prime}$ plane. They established the limits of quintessence model in phase space of $\omega_{\vartheta}$ and $\omega_{\vartheta}^{\prime}$ and pointed 

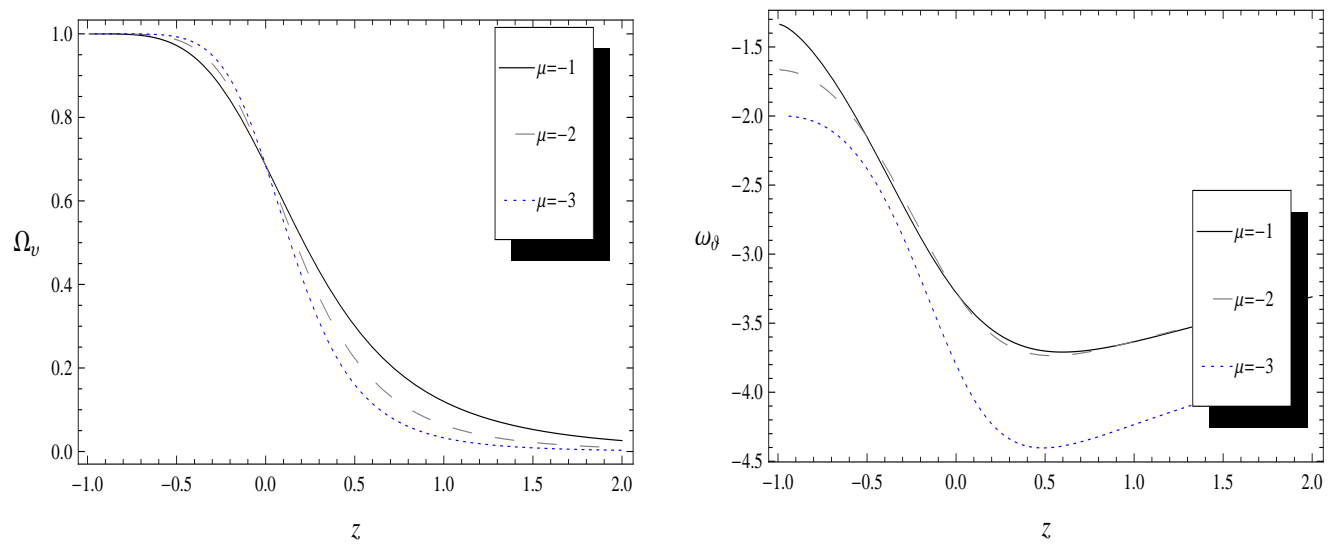

Figure 2: Evolution of $\Omega_{\vartheta}$ and $\omega_{\vartheta}$ for PDE with particle horizon $(\mu \leqslant-1)$.

out two regions of this plane namely thawing $\left(\omega_{\vartheta}^{\prime}>0\right.$ with $\left.\omega_{\vartheta}<0\right)$ and freezing $\left(\omega_{\vartheta}^{\prime}<0\right.$ with $\left.\omega_{\vartheta}<0\right)$. It is remarked that cosmic expansion is accelerated in freezing region when compared with thawing region. This approach has been applied in different settings by considering various forms of DE such as quintessence, phantom and quintom models (Chiba 2006; Gao et al. 2006; Scherrer 2006). In (Sharif and Zubair 2013f), we have also discussed the phase space of $\omega_{\vartheta}$ and $\omega_{\vartheta}^{\prime}$ for new HDE which exhibits $\Lambda$ CDM model $\left(\omega_{\vartheta}=-1\right.$ and $\left.\omega_{\vartheta}^{\prime}=0\right)$ in future evolution. The evolution of $\omega_{\vartheta}^{\prime}$ in $\omega_{\vartheta}-\omega_{\vartheta}^{\prime}$ plane is shown in the right panel of Figure 3. This represents the freezing region for noninteracting PDE with particle horizon which favors the phantom evolution in this format of DE.

In what follows, we examine PDE with particle horizon using the statefinder diagnostic. The pair $\{r, s\}$ of statefinder diagnostic parameters is defined as (Sahni et al. 2003)

$$
r=\frac{\dddot{a}}{a H^{3}}, \quad s=\frac{(r-1)}{3(q-1 / 2)},
$$

$r$ is also named as jerk parameter. Statefinder diagnostic depends on the scale factor, its derivatives $\dddot{a}$ and deceleration parameter $q$ to differentiate the cosmic expansion on geometric grounds. Cosmological models can be differentiated on the basis of statefinder diagnostic as it shows distinct trajectories corresponding to specific models. For $\Lambda$ CDM model, the statefinder parameters are fixed as $(r, s)=(1,0)$ and in case of CDM regime these correspond to $(r, s)=(1,1)$. In $r-s$ plane, the trajectories for quintessence 

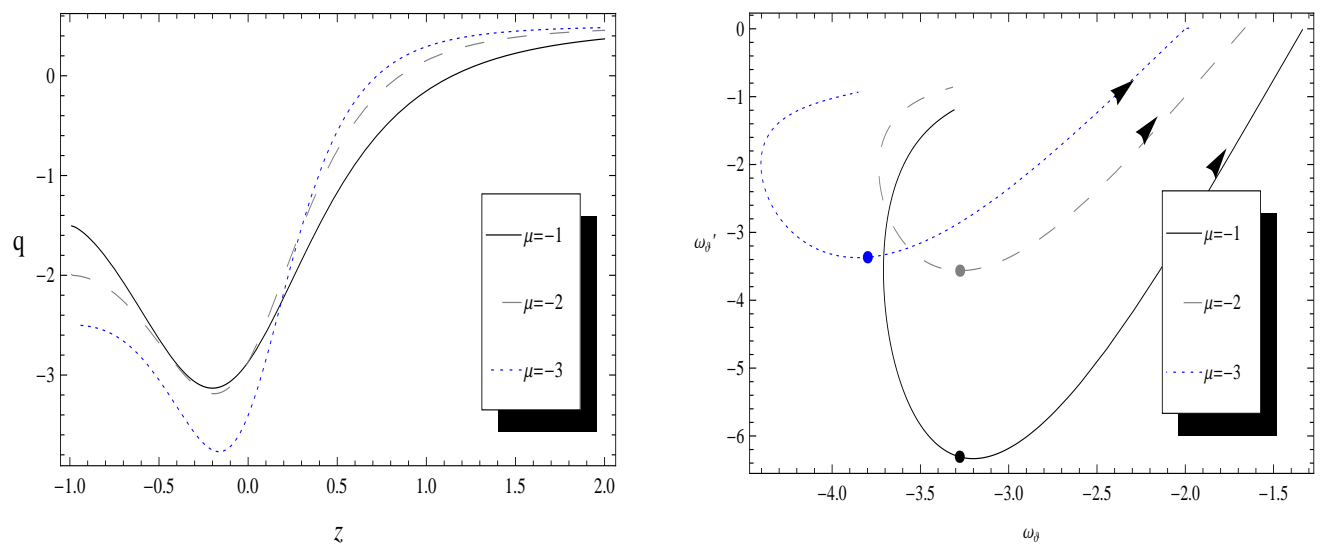

Figure 3: Evolution of $q$ and $\omega_{\vartheta}-\omega_{\vartheta}^{\prime}$ plane for PDE with particle horizon for $\mu \leqslant-1$. A sign flip of $q$ indicates transition to accelerated expansion and $\omega_{\vartheta}-\omega_{\vartheta}^{\prime}$ plane indicates the freezing region. The dots represent present day values of parameters.

and phantom lie in the range $(s>0, r<1)$ whereas for chaplygin gas these correspond to $(s<0, r>1)$. The statefinder diagnostic parameters can be represented in terms of $\omega_{\vartheta}$ and $\Omega_{\vartheta}$ as

$$
r=1-\frac{3}{2} \Omega_{\vartheta}\left[\omega_{\vartheta}^{\prime}-3 \omega_{\vartheta}\left(1+\omega_{\vartheta}\right)\right], \quad s=\frac{-1}{3 \omega_{\vartheta}}\left[\omega_{\vartheta}^{\prime}-3 \omega_{\vartheta}\left(1+\omega_{\vartheta}\right)\right] .
$$

Figure 4 shows the evolution trajectories of PDE with particle horizon in $r-s$ and $q-r$ planes. In the left panel, the evolution trajectories favor the chaplygin gas model with $s<0$ and $r>1$. We also plot the evolution trajectories of the deceleration parameter in $q-r$ plane. Our results are consistent with (Wu and Yu 2005, 2006) where authors performed the statefinder diagnostic for the phantom and quintom DE model. This shows that the noninteracting PDE with particle horizon favors the phantom regime which is the basic idea of this candidate. Thus, for the realistic model of PDE, one needs to set $\mu<0$ and this choice is well supported by the results of Planck and WMAP9 observations (Ade et al. 2013; Bennet 2012). Here we take $\mu \leqslant-1$ for which PDE implies that $\omega_{\vartheta}<-1$ which is supported by other cosmographic parameters as shown in Figures $\mathbf{3}$ and $\mathbf{4}$. 

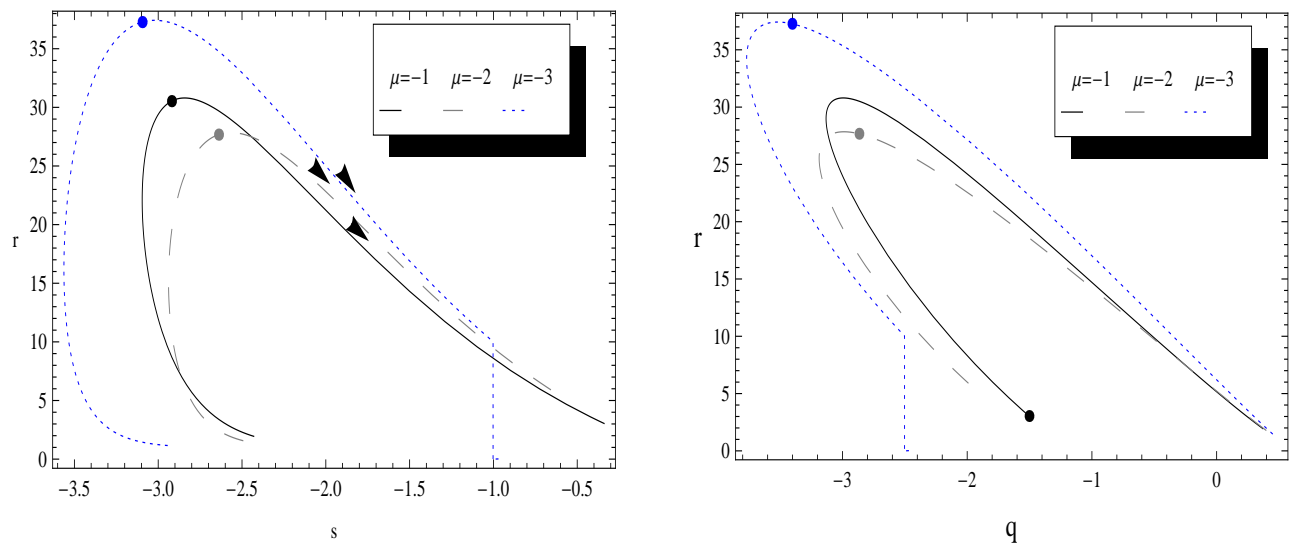

Figure 4: Evolution of statefinder diagnostic parameters for PDE with particle horizon $(\mu \leqslant-1)$.

\subsection{Event Horizon}

The IR cutoff $L$ (event horizon) is defined as

$$
L=R_{\hat{E}}=a(t) \int_{t}^{\infty} \frac{d \hat{t}}{a(\hat{t})}=a(t) \int_{a}^{\infty} \frac{d a^{\prime}}{H a^{\prime 2}} .
$$

Employing the definition of PDE (11) and event horizon (12), we obtain the dynamical equation of fractional density of DE as

$$
\Omega_{\vartheta}^{\prime}=\Omega_{\vartheta}\left(1-\Omega_{\vartheta}\right)\left(3-\mu+\frac{\mu}{C}\left(1-\Omega_{\vartheta}\right)^{1 / 2-1 / \mu}\left(\Omega_{\vartheta}\right)^{1 / \mu} e^{(3 / 2-3 / \mu) x}\right) .
$$

The time derivative of PDE with event horizon as an IR cut-off is

$$
\dot{\rho}_{\vartheta}=-\mu \rho_{\vartheta} \frac{\dot{L}}{L}, \quad \dot{L}=H L-1 .
$$

Using the energy conservation equation of DE, we obtain

$$
\omega_{\vartheta}=-1+\frac{\mu}{3}\left(1-\frac{1}{H}\left(\frac{H^{2} \Omega_{\vartheta}}{n^{2} M_{p}^{2-\mu}}\right)^{1 / \mu}\right) .
$$

One can reproduce the corresponding results in HDE with event horizon for $\mu=2$. In case of HDE with event horizon, the EoS parameter is $\omega_{\vartheta}=$ 
(a)

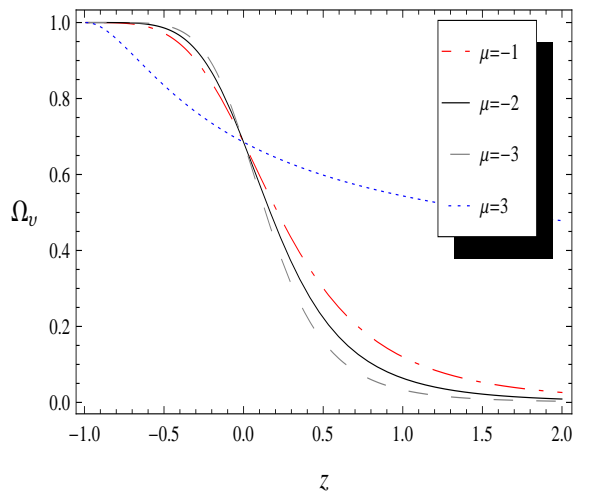

(b)

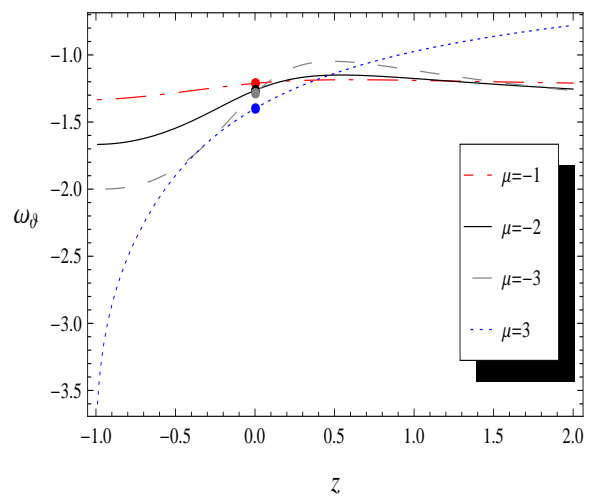

Figure 5: Evolution of $\Omega_{\vartheta}$ and $\omega_{\vartheta}$ versus $z$ for PDE with event horizon for $n=0.5$ and different values of $\mu$. The dots indicate the present day values.

$-\frac{1}{3}\left(1+\frac{2 \sqrt{\Omega_{\vartheta}}}{n}\right)$ which can result in three significant eras of cosmic expansion. If the universe is dominated by DE components, i.e., $\Omega_{\vartheta} \longrightarrow 1$ in the future then for $n>1, \omega_{\vartheta}$ is always greater than -1 which depicts quintessence era so that the universe escapes from getting in de Sitter and big rip phases. For $n=1$, the universe enters the de Sitter era in future evolution and $n<1$ represents phantom phase where the universe behaves as quintom model of $\mathrm{DE}$ as $\omega_{\vartheta}$ intersects the cosmological constant line. The value of $n$ plays a vital role in deciding the evolutionary features of HDE and ultimate fate of the universe.

For PDE, the role of $\mu$ is more crucial as compared to that of $n$. In this setting, we are mainly concerned with the choice $\mu<0$ but for PDE with event horizon, one can also set $\mu=3$. The evolution trajectories of EoS and fractional energy density of PDE are shown in Figure 5. For $\mu \leqslant-1$, $\Omega_{\vartheta}$ approaches to 1 as $z \rightarrow-1$ showing that DE dominates in later times of the universe. The EoS parameter $\omega_{\vartheta}$ is in the phantom regime (Figure $5(\mathbf{b}))$ and the present day values of $\omega_{\vartheta}$ are consistent with the Planck results showing $\omega_{\vartheta}=-1.13_{-0.10}^{+0.13}$ (Bennet 2012). In case of $\mu>2$, we show the evolution trajectories for $\mu=3$ which does favor the phantom feature of DE. For $\mu=3$, the curves in Figure $\mathbf{5}$ show somewhat distinct behavior where $\omega_{\vartheta}<-3.5$ as $z \rightarrow-1$. We neglect the values of $\mu>3$ because these values do not imply realistic results. The evolution of $q$ versus $z$ is represented in Figure 6(a) which shows that the universe entered into the accelerating 
(a)

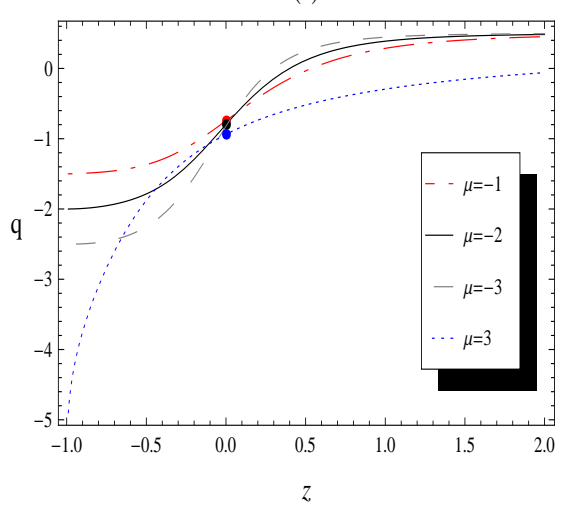

(b)

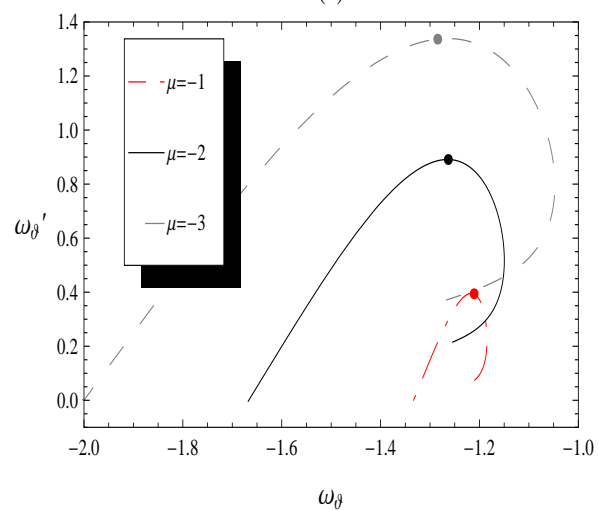

Figure 6: Plot 6(a) shows the variation of $q$ versus $z$ and 6(b) represents evolution trajectories of $\omega_{\vartheta}-\omega_{\vartheta}^{\prime}$ for non-interacting PDE with event horizon corresponding to $n=0.5$ and different values of $\mu$. The dots indicate the present day values of $q$ and $\left\{\omega_{\vartheta}, \omega_{\vartheta}^{\prime}\right\}$.

phase in the recent past and it would switch over to $q<-1$ indicating the phantom paradigm.

Taking derivative of Eq.(14) with respect to $x$, we get

$$
\omega_{\vartheta}^{\prime}=\left(1-\frac{3}{\mu}\left(1+\omega_{\vartheta}\right)\right)\left(1-\frac{\mu}{2}-\frac{1}{3} \frac{d}{d x} \ln \Omega_{\vartheta}\right) .
$$

The phase plane of $\omega_{\vartheta}$ and $\omega_{\vartheta}^{\prime}$ for non-interacting PDE with event horizon is shown in Figure 6(b). The plane $\omega_{\vartheta}-\omega_{\vartheta}^{\prime}$ represents the thawing region in the evolution of PDE with event horizon. In the limit of future evolution $z \rightarrow-1, \omega_{\vartheta}^{\prime} \rightarrow 0$ and $\omega_{\vartheta}>-1$. Figure 7 shows statefinder analysis in $q-r$ plane for the choice $\mu \leqslant-1$. The evolution trajectories in $q-r$ plane for PDE with event horizon start from $(q<0.5, r<1)$ and end up with $(q<-1, r>1)$.

\subsection{Conformal Age of the Universe}

The two time scales, age of the universe and conformal time have been suggested in literature corresponding to agegraphic DE (ADE) (Cai 2007) and new agegraphic DE (NADE) (Wei and Cai 2008a). These models can derive the cosmic expansion consistent with the recent observational data (Wei and 

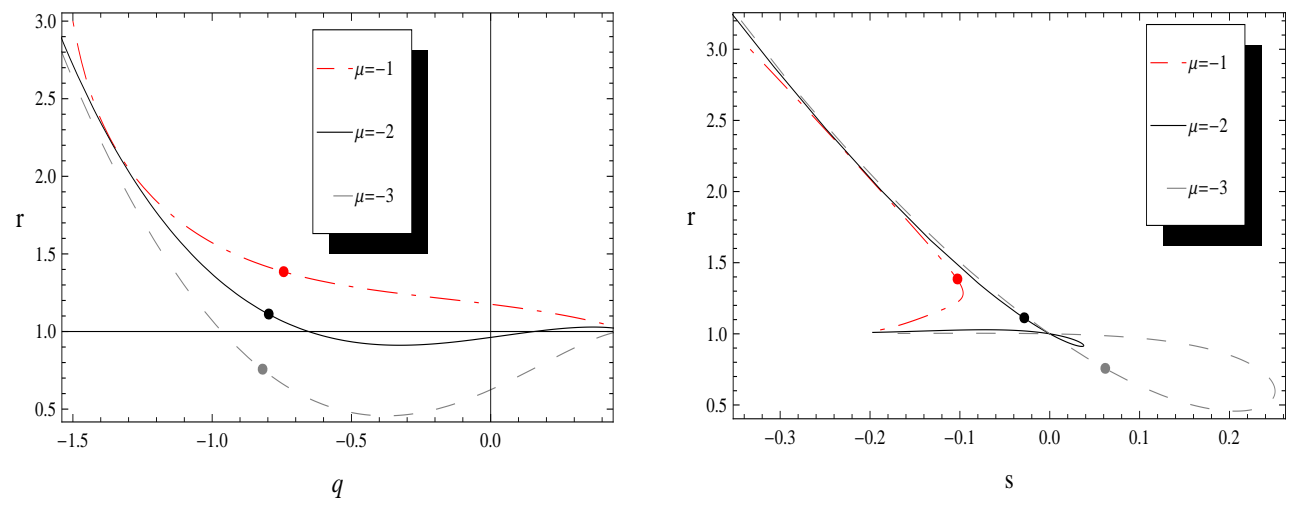

Figure 7: The statefinder analysis of PDE with event horizon in $q-r$ and $r-s$ planes.

Cai 2008b) which can resolve the causality problem. However, it is pointed out that ADE model is classically unstable and NADE is no better than HDE in explaining the DE dominated universe. The NADE has been studied to address various cosmological issues in Einstein and modified gravities (Karami 2010; Jamil and Saridakis 2010; Sheykhi 2010). The conformal age of the universe is defined as

$$
\eta=\int \frac{d t}{a(t)}=\int \frac{d a}{H a^{2}} .
$$

Using Eqs.(1) and (16), the rate of change of fractional DE density is

$$
\Omega_{\vartheta}^{\prime}=\Omega_{\vartheta}\left(1-\Omega_{\vartheta}\right)\left(3-\mu-\frac{\mu}{C}\left(1-\Omega_{\vartheta}\right)^{1 / 2-1 / \mu}\left(\Omega_{\vartheta}\right)^{1 / \mu} e^{(1 / 2-3 / \mu) x}\right) .
$$

The time derivative of $\rho_{\vartheta}$ is obtained as

$$
\dot{\rho}_{\vartheta}=-\frac{\mu \rho_{\vartheta}}{a}\left(\frac{H^{2} \Omega_{\vartheta}}{n^{2} M_{p}^{2-\mu}}\right)^{1 / \mu} .
$$

The corresponding EoS parameter is

$$
\omega_{\vartheta}=-1+\frac{\mu}{3 a H}\left(\frac{H^{2} \Omega_{\vartheta}}{n^{2} M_{p}^{2-\mu}}\right)^{1 / \mu} .
$$

To demonstrate the evolution trajectories for NADE version of PDE, we include some facts about non-interacting NADE model. Wei and Cai (2008b) 
showed that coincidence problem can be resolved for NADE if one chooses the value of parameter $n$ nearly unity. The NADE is constrained from the observational data of SNeIa, CMB and LSS which implies the best fit value

of $n=2.76_{-0.109}^{+0.111}$ (with $1 \sigma$ uncertainty). They found that EoS parameter for NADE approaches to -1 in later times regardless of the value of $n$. Zhang et al. (2013) showed WMAP 7-years observations set appropriate measure of $n$ as $n=2.673_{-0.077-0.151-0.222}^{+0.053+0.127+0.199}$. In previous study (Sharif and Zubair 2013f), we have reconstructed $f(R)$ gravity corresponding to NADE and set the values of $n$ as $n=2.3,2.8,3.3,3.8$. It has been shown that these parametric values support the $\Lambda \mathrm{CDM}$ model in future evolution.

For PDE with conformal time scale, we are mainly concerned to explore the behavior of parameter $\mu$ in determining the evolution for conformal time. Initially, we set $\mu<0$ and found that $\mu=-1$ supports the cosmological constant regime in future evolution as shown in Figures 8 and 9. Figure 8(a) shows that $\Omega_{\vartheta} \rightarrow 1$ in the future evolution so that our universe is dominated by DE. $\omega_{\vartheta}$ is always less than -1 in the whole cosmic history and it will asymptotically approach to -1 in the future evolution of the universe. The present behavior of EoS parameter favors the phantom DE consistent with Planck and WMAP9 results. Therefore, evolution of the universe will end up with $\Lambda$ CDM model rather than big rip. Such behavior is identical to that suggested by Wei and Cai (2008b) for PDE with Hubble horizon. In Figure 9(a), the evolution of deceleration parameter shows the bouncing behavior of the universe which entered in the expansion phase in recent past and it would end up in a de Sitter phase.

If we differentiate Eq.(18) with respect to $x$, we have

$$
\omega_{\vartheta}^{\prime}=-e^{-x}\left(1-\frac{3}{\mu}\left(1+\omega_{\vartheta}\right)\right)\left(1-\frac{\mu}{6}-\frac{1}{3} \frac{d}{d x} \ln \Omega_{\vartheta}\right) .
$$

Figure $\mathbf{9}(\mathbf{b})$ shows the evolution trajectories of $\omega_{\vartheta}^{\prime}$ in $\omega_{\vartheta}-\omega_{\vartheta}^{\prime}$ plane for PDE with cosmological time scale. It is obvious that evolution in phase space of $\omega_{\vartheta}$ and $\omega_{\vartheta}^{\prime}$ results in $\Lambda$ CDM model $\left(\omega_{\vartheta}=-1, \omega_{\vartheta}^{\prime}=0\right)$ as $z \rightarrow-1($ or $x \rightarrow \infty)$. We analyze that if one sets $-55<\mu<-1$, it would result in $\omega_{\vartheta} \rightarrow 0$ (i.e., the matter dominated universe) in future evolution. If one chooses $\mu \leqslant-55$, we can have $\omega_{\vartheta}>-1$ in later times of cosmic evolution but in such case the present day value is only consistent with WMAP9 observational results as it represents the quintessence model of DE (see Figure 10). For PDE with cosmological time scale, we also present the evolution trajectories for $\mu \geqslant 3$ 
(a)

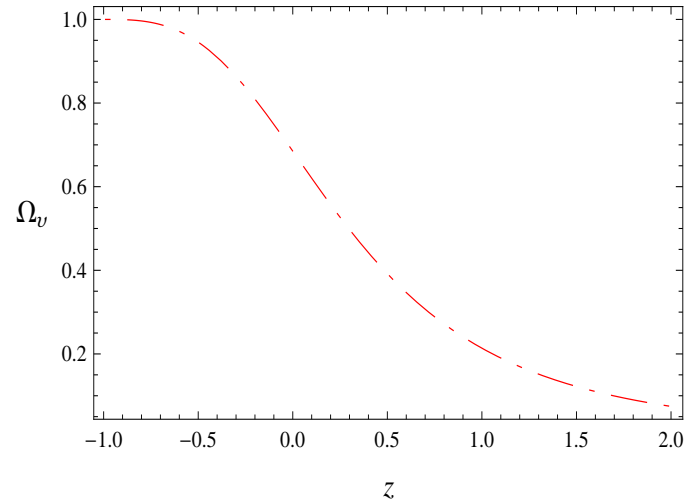

(b)

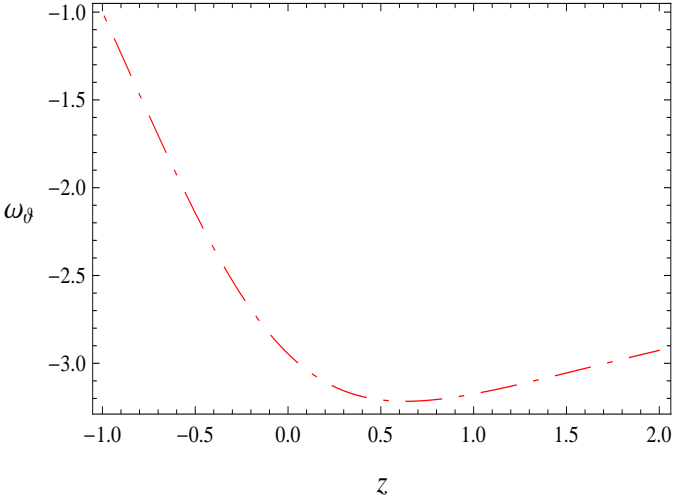

Figure 8: Evolution trajectories of $\Omega_{\vartheta}$ and $\omega_{\vartheta}$ for PDE with conformal time. We set $\mu=-1$ and $n=2$.

(a)

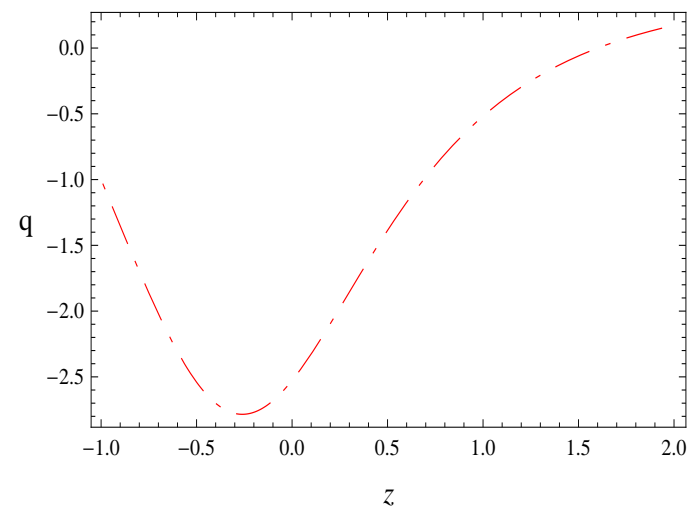

(b)

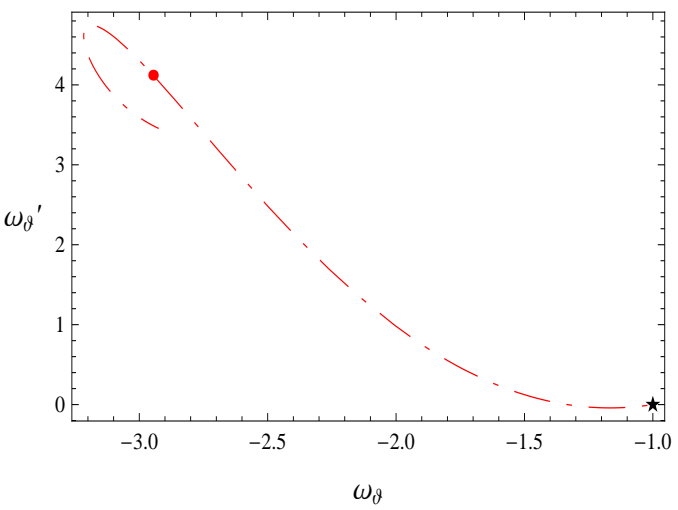

Figure 9: Evolution trajectories of $q$ and $\omega_{\vartheta}-\omega_{\vartheta}^{\prime}$ phase plane for $\mu=-1$ and $n=2$. Star indicates the $\Lambda$ CDM model with $\omega_{\vartheta}=-1$ and $\omega_{\vartheta}^{\prime}=0$. 
(a)

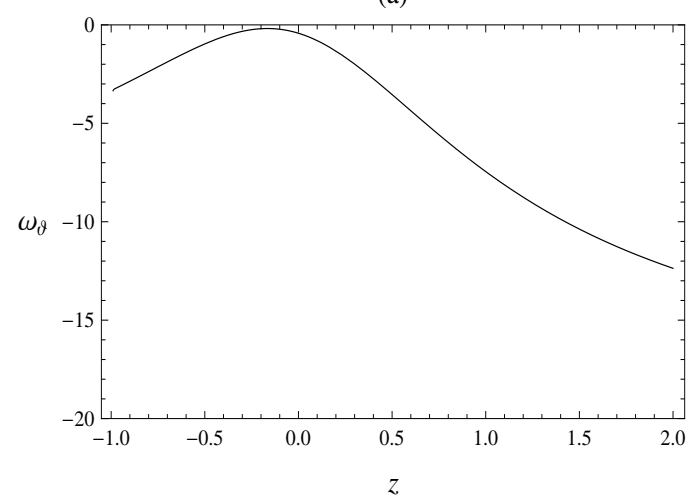

(b)

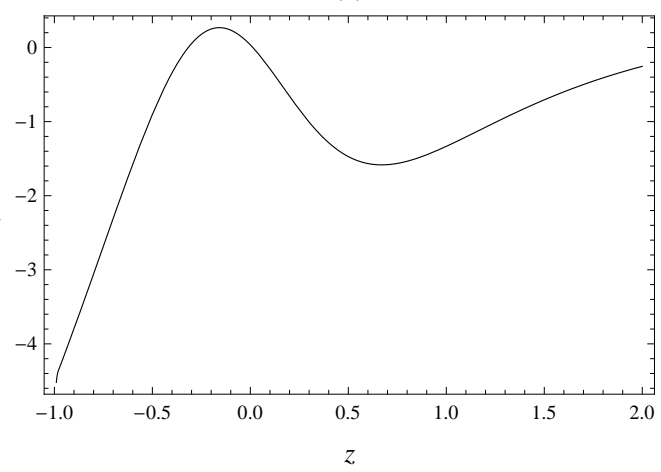

Figure 10: Evolution trajectories of $\omega_{\vartheta}$ and $q$ versus redshift for PDE with conformal time corresponding to $\mu=-55$ and $n=2$.

as shown in Figure 11. It is found that for $\mu=3$, we have $\Lambda$ CDM regime whereas for $\mu \geqslant 4$, it results in quintessence era of the universe.

\section{Conclusions}

In this paper, we study the phantom evolution of PDE with three cut-offs namely particle horizon, event horizon and conformal age of the universe in FRW spacetime. We explore these IR cut-offs to establish the consistent range in PDE for parameter $\mu$ and also the phantom regime. Following (Li 2004), the evolution equation of fractional energy density of DE $\Omega_{\vartheta}$ and dynamical relation of $\omega_{\vartheta}$ are formulated in these settings. If $\mu=2$, one can determine the corresponding results in HDE with particle horizon, event horizon and conformal age of the universe. We set the present day values of parameters according to recent Planck observations and present the evolution inconsistent with this data set.

Firstly, we have analyzed the non-interacting PDE with particle horizon and shown the evolutionary paradigm of $\Omega_{\vartheta}$ and $\omega_{\vartheta}$. If $\mu \geqslant 3$, then we have purely matter dominated phase of the universe since $\Omega_{\vartheta} \rightarrow 0$ and $\omega_{\vartheta}>1$ for $z \rightarrow-1$ as shown in Figure 1. This choice is neglected in search of some consistent models. For $\mu<0$, the plots of $\Omega_{\vartheta}$ and $\omega_{\vartheta}$ are shown in Figure 2 which represents that $\omega_{\vartheta}$ is in the phantom regime and never intersects the phantom divide line in the whole cosmic history. This is identical to 
(a)

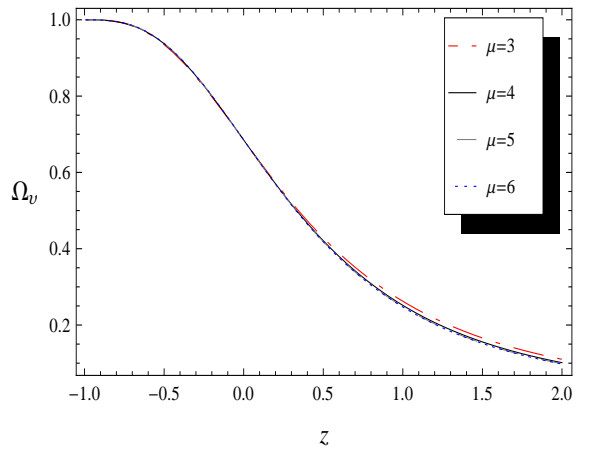

(b)

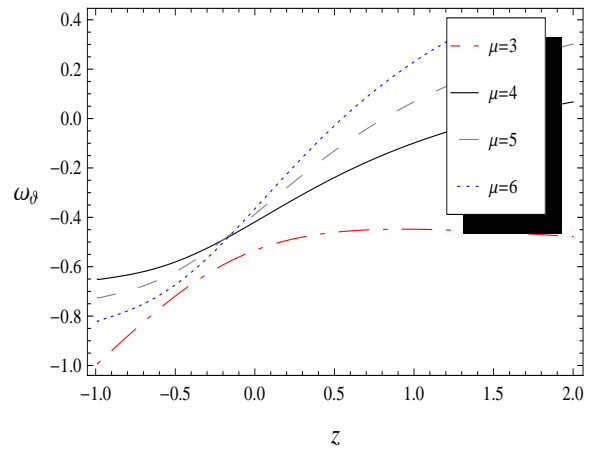

(c)

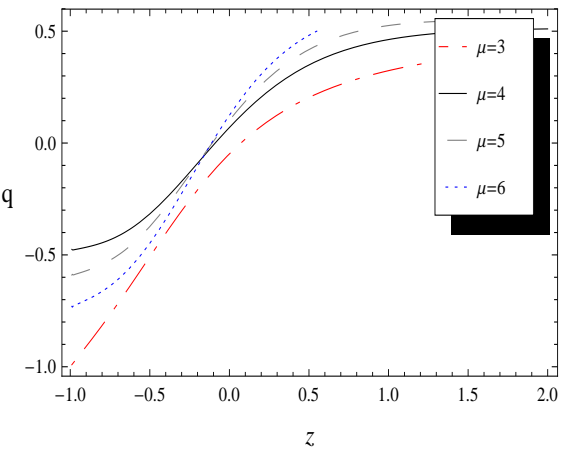

(d)

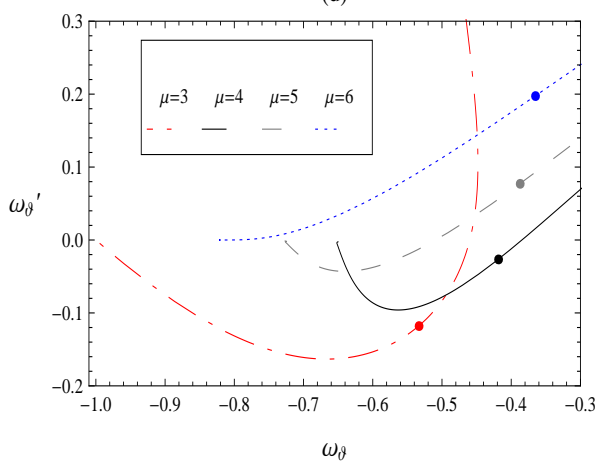

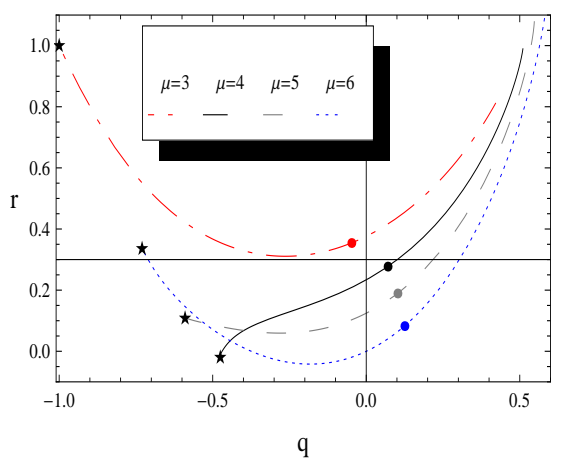

Figure 11: Evolution trajectories of $\Omega_{\vartheta}, \omega_{\vartheta}$ and $q$ versus $z$ and in $\omega_{\vartheta}-\omega_{\vartheta}^{\prime}$ and $q-r$ planes for PDE with conformal time. Plot (a) shows the evolution trajectories of $\Omega_{\vartheta}$ which results in DE dominated era in future evolution. In plot (b) it is evident that for $\mu=3$, we have de Sitter phase whereas $\mu \geqslant 4$ represents the quintessence regime. This behavior is well supported by the evolution of $q$ in plot (c). The evolution trajectories of $\omega_{\vartheta}^{\prime}$ are shown in plot (d) which indicate the $\Lambda \mathrm{CDM}\left(\omega_{\vartheta}, \omega_{\vartheta}^{\prime}\right)=(-1,0)$ model for $\mu=3$. Statefinder diagnosis can also be seen in plot (e). 
that of PDE with Hubble horizon (Wei 2012). The evolution of $\omega_{\vartheta}$ shows the phantom regime inconsistent with the current observational results of Planck and WMAP9 data sets. We also plot deceleration parameter and the phase space of $\omega_{\vartheta}$ and $\omega_{\vartheta}^{\prime}$ in Figure 3. $\omega_{\vartheta}^{\prime}$ lies in the freezing region favoring the phantom evolution in this format of DE. The statefinder diagnostic (Figure 4) show that evolution trajectories are consistent with phantom regime ( $\mathrm{Wu}$ and $\mathrm{Yu} 2005,2006)$. Thus, we conclude that for realistic model of PDE with particle horizon one needs to set $\mu<0$ and this choice is well supported by the results of Planck and WMAP9 observations.

Secondly, we have explored PDE scenario in the light of event horizon. We are mainly concerned with the choice of $\mu<0$ but for PDE with event horizon one can also set $\mu=3$. For $\mu \leqslant-1$ and $\mu=3$, the evolution of $\omega_{\vartheta}$ and $\Omega_{\vartheta}$ is shown in Figure 5. It is found that DE dominates in future evolution as $\Omega_{\vartheta} \rightarrow 1$ for $z$ approaching to -1 . The EoS parameter is in the quintessence regime in recent past which bisects the phantom divide line and ends up in phantom era. For $\mu>2$, the acceptable results are found only for $\mu=3$ whereas parameter $\mu>3$ does not show realistic results. Figure 6(a) favors the phantom DE showing sign flip of $q$ in recent past leading to $q<-1$. We also show the evolution trajectories in $\omega_{\vartheta}-\omega_{\vartheta}^{\prime}$ plane (Figure $\mathbf{6}(\mathbf{b})$ ). It represents the thawing region and in later times of the universe $\omega_{\vartheta}^{\prime} \rightarrow 0$ with $\omega_{\vartheta}>-1$. These results are also presented in $q-r$ plane (Figure 7). It is found that for PDE with event horizon the acceptable range of $\mu$ is $\mu \leqslant-1$ and $\mu=3$.

Thirdly, we have used conformal time scale as IR cut-off for PDE. Initially, we set $\mu=-1$ and plot the fractional density and EoS parameter of DE (Figure 8). Accordingly, $\Omega_{\vartheta} \rightarrow 1$ shows the dominance of DE and $\omega_{\vartheta}<-1$ in entire cosmic evolution which approaches to -1 in ultimate fate of the universe. Consequently, cosmic evolution ends up with cosmological constant regime avoiding the big rip singularity which is identical to Wei (2012) for Hubble horizon. The deceleration parameter $q$ shows the bouncing behavior of the universe where the universe entered in accelerated expansion era in recent past and concludes in de Sitter phase. These results are also favored by the phase space of $\omega_{\vartheta}$ and $\omega_{\vartheta}^{\prime}$ as shown in Figure $\mathbf{9}(\mathbf{b})$. It is found that the range $-55 \leqslant \mu<-1$ results in matter dominated cosmic evolution. If one sets $\mu \leqslant-55, \omega_{\vartheta}$ can show phantom evolution for later times but in such case the present day value of $\omega_{\vartheta}$ is not observationally consistent. Hence, for conformal age of the universe in PDE scenario, the only acceptable value of $\mu$ is -1 . 
In (Sharif and Jawad 2013), authors discussed the PDE for non-interacting case by defining EoS and other cosmographic parameters in terms of present day values of $\Omega_{\vartheta}, \Omega_{m}$ and $H$. This study is confined to present scenario and does not show the behavior in entire cosmic evolution. Comparatively, the dynamical equation of $\Omega_{\vartheta}$ with the initial condition $\Omega_{\vartheta 0}=1-\Omega_{m 0}$ implies that the entire cosmic evolution of $\Omega_{\vartheta}$ as well as $\omega_{\vartheta}$ can be established. We remark that PDE with particle horizon leads to phantom evolution if $\mu<0$ and for event horizon one can set both $\mu<0$ and $\mu=3$. In case of conformal age of the universe, the result is quite significant where only consistent value is $\mu=-1$ showing identical behavior to that for the Hubble horizon (Wei 2012).

\section{Acknowledgment}

We would like to thank the Higher Education Commission, Islamabad, Pakistan for its financial support through the Indigenous Ph.D. 5000 Fellowship Program Batch-VII.

\section{References}

Armendariz-Picon, C., Mukhanov, V.F., Steinhardt, P.J.: Phys. Rev. Lett. 85, 4438(2000)

Arkani-Hamed, N., Cheng, H.C., Luty, M.A., Mukohyama, S.: J. High Energy Phys. 07, 065(2002)

Babichev, E. Dokuchaev, V., Eroshenko, Y.: Phys. Rev. Lett. 93, 0211102(2004)

Bennet, C.L.: arXiv:1212.5225v3

Cai, R.-G.: Phys. Lett. B 657, 228(2007)

Caldwell, R.R.: Phys. Lett. B 545, 23(2002)

Caldwell, R.R., Linder, E.V.: Phys. Rev. Lett. 95, 141301(2005)

Chiba, T.: Phys. Rev. D 73, 063501(2006)

Cohen, A.G., Kaplan, D.B., Nelson, A.E.: Phys. Rev. Lett. 82, 4971(1999)

Feng, B., Wamg, X.L., Zhang, X.M.: Phys. Lett. B 607, 35(2005)

Fischler, W., Susskind, L.: arXiv:hep-th/9806039

Gao, C., Wu, F., Chen, X., Shen, Y.-G.: Phys. Rev. D 79, 043511(2009) Gao, C., Chen, X., Faraoni, V., Shen, Y.G.: Phys. Rev. D 78, 
024008(2008)

Gao, Z.K., Piao, Y.S., Zhang, X.M., Zhang, Y.Z.: Phys. Rev. D 74, 127304(2006)

Gonzalez, J.A., Guzman, F.S.: Phys. Rev. D 79, 121501(2009)

Granda, L.N., Oliveros, A.: Phys. Lett. B 669, 275(2008)

Granda, L.N., Oliveros, A.: Phys. Lett. B 671, 199(2009)

Haghani, Z. et al.: Phys. Rev. D 88, 044023(2013)

Harko, T., Lobo, F.S.N., Nojiri, S., Odintsov, S.D.: Phys. Rev. D 84, 024020(2011)

Hsu, S.D.H.: Phys. Lett. B 594, 13(2004)

Jamil, M., Qadir, A.: Gen. Rel. Grav. 43, 1069(2011)

Jamil, M., Saridakis, E.N.: J. Cosmol. Astropart. Phys. 07, 028(2010)

Jassal, H.K., Bagla, J.S., Padmanabhan, T.: Mon. Not. R. Astron. Soc. 405, 2639(2010)

Karami, K.: Phys. Lett. B 686, 216(2010)

Li, M.: Phys. Lett. B 603, 1(2004)

Peebles, P.J.E., Ratra, B.: Rev. Mod. Phys. 75, 559(2003)

Perlmutter, S. et al.: Astrophys. J. 517, 565(1999)

Planck collaboration, Ade, P. et al.: arXiv:1303.5062

Riess, A.G. et al.: Astrophys. J. 659, (2007)98

Sahni, V., Saini, T.D., Starobinsky, A.A., Alam, U.: J. Exp. Theor. Phys. Lett. 77, 201(2003)

Samushia, L., Ratra, B.: Astrophys. J. 680, L1(2008)

Scherrer, R.J.: Phys. Rev. D 73, 043502(2006)

Sharif, M., Abbas, G.: Chin. Phys. Lett. 28, 090402(2011)

Sharif, M., Abbas, G.: Chin. Phys. Lett. 29, 010401(2012)

Sharif, M., Zubair, M.: J. Cosmol. Astropart. Phys. 03(2012a)028

[Erratum-ibid 05, E01(2012)]

Sharif, M., Zubair, M.: J. Phys. Soc. Jpn. 81, 114005(2012b)

Sharif, M., Zubair, M.: J. Exp. Theor. Phys. 117, 248(2013a)

Sharif, M., Zubair, M.: J. Phys. Soc. Jpn.82, 014002(2013b)

Sharif, M., Zubair, M.: J. Phys. Soc. Jpn. 82, 064001(2013c)

Sharif, M., Zubair, M.: J. Cosmol. Astropart. Phys. 11, 042(2013d)

Sharif, M., Zubair, M.: J. High Energy Phys. 12, 079(2013e)

Sharif, M., Zubair, M.: Adv. High Energy Phys. 2013, 790967(2013f)

Sharif, M., Jawad, A.: Eur. Phys. J. C 73, 2382(2013)

Sheykhi, A.: Phys. Rev. D 81, 023525(2010)

Sotiriou, T.P., Faraoni, V.: Rev. Mod. Phys. 82, 451(2010) 
Spergel, D.N. et al.: Astrophys. J. Suppl. Ser. 170, 377(2007)

Steinhardt, P.J., Wang, L., Zlatev, I.: Phys. Rev. D 59, 123504(1999)

Sun, Y.C.: Commun. Theor. Phys. 52, 441(2009)

Weinberg, S.: Rev. Mod. Phys. 61, 1(1989)

Wei, H.: Class. Quantum Grav. 29, 175008(2012)

Wei, H., Cai, R.-G.: Phys. Lett. B 660, 113(2008a)

Wei, H., Cai, R.-G.: Phys. Lett. B 663, 1(2008b)

Wu, P., Yu, H.: Int. J. Mod. Phys. D 14, 1873(2005)

Wu, P., Yu, H.: Mod. Phys. Lett. A 21, 1305(2006)

Tegmark, M. et al.: Phys. Rev. D 69, 103501(2004)

Zhang, J.-F., Li, Y.-H., Zhang, X.: Eur. Phys. J. C 73, 2280(2013) 